\title{
Efficacy and safety of Qi-Wei-Qing-Yan aerosol in treatment of acute pharyngitis (lung-stomach excess-heat syndrome): study protocol for a randomized controlled trial
}

Hong-li Jiang ${ }^{1}$, Bin She', Wei Liu', Bing Mao ${ }^{1 *}$ and Ju-ying Zhang ${ }^{2}$

\begin{abstract}
Background: Acute pharyngitis accounts for an estimated 15 million patient visits in the United States. However, there is no proven effective and safe treatment. Although Chinese herbal medicine is widely used in the treatment of acute pharyngitis, there is a lack of evidence-based data. Despite several clinical trials conducted in this setting, no randomized placebo-controlled trial has been performed to date. This trial aims to investigate the efficacy and safety of Qi-Wei-Qing-Yan aerosol (QWQYA), a Chinese herbal prescription, compared with a placebo aerosol in the treatment of acute pharyngitis with lung-stomach excess-heat syndrome.
\end{abstract}

Methods/design: This is a prospective, multicenter, randomized, double-blinded, parallel-group, placebo-controlled trial. A total of 420 adult patients, of either sex, with acute pharyngitis will be enrolled from seven study sites across China. All patients will be randomly allocated to one of three parallel treatment groups: (1) QWQYA with the current propellant, (2) QWQYA with a previous propellant, and (3) the placebo aerosol with the current propellant. The study medication will be administered into the pharyngeal region in three sprays thrice daily for 5 consecutive days.

The primary outcome measures are time to complete resolution of sore throat and relief rate of sore throat. Secondary outcome measures include resolution rate of sore throat, time to relief of sore throat, intensity of sore throat, and change of traditional Chinese medicine syndrome score and clinical signs score from baseline to post-treatment, as well as the occurrence of any adverse events.

Discussion: This will be the first clinical trial to investigate the efficacy and safety of QWQYA in the treatment of acute pharyngitis in an adult population in a multicenter, randomized, double-blinded, parallel-group, placebo-controlled manner. Not only might it establish the basis for the efficacy and safety of QWQYA in treating acute pharyngitis, but it might also provide evidence to support the use of Chinese herbal medicine in treating acute pharyngitis and thus support an alternative treatment option for management of acute pharyngitis.

Trial registration: Chinese Clinical Trial Registry ChiCTR-IPR-15005991.

Keywords: acute pharyngitis, Qi-Wei-Qing-Yan aerosol, randomized controlled trial, sore throat, traditional Chinese medicine

\footnotetext{
* Correspondence: maobing@medmail.com.cn

${ }^{1}$ Department of Integrated Traditional Chinese and Western Medicine, West

China Hospital, Sichuan University, No. 37 Guoxue Street, Chengdu 610041,

Sichuan Province, China

Full list of author information is available at the end of the article
}

\section{) Biomed Central}

(c) 2016 Jiang et al. Open Access This article is distributed under the terms of the Creative Commons Attribution 4.0 International License (http://creativecommons.org/licenses/by/4.0/) which permits unrestricted use, distribution, and reproduction in any medium, provided you give appropriate credit to the original author(s) and the source, provide a link to the Creative Commons license, and indicate if changes were made. The Creative Commons Public Domain Dedication waiver (http://creativecommons.org/publicdomain/zero/1.0/) applies to the data made available in this article, unless otherwise stated. 


\section{Background}

Acute pharyngitis is characterized by an inflammation of mucous membranes of the pharynx and the surrounding lymphoid tissue and accounts for an estimated 15 million patient visits in 2006 in the United States [1]. It is commonly caused by various viral or bacterial infections. Viruses are the most common cause, accounting for up to $40 \%$ to $60 \%$ of cases [2]. The most common type of bacterial infection is group A beta-hemolytic streptococcal (GABHS) infection, which is responsible for around one-third of pediatric pharyngitis but only $5 \%$ to $10 \%$ of adult pharyngitis [3, 4]. An acute onset of sore throat is the predominant symptom, accounting for more than $10 \%$ of all primary care visits [1].

Antibiotics are indicated to prevent suppurative complications and rheumatic fever, and decrease infectivity, but may only provide limited benefit in the resolution of symptoms, shortening sore throat duration by less than 1 day [5]. Moreover, excessive or inappropriate use of antibiotics may be associated with the potential side effects, increased antibacterial drug resistance, and high medical costs [6, 7]. Given that the majority of cases are caused by viral pathogens, the current treatment strategy in adults focuses on the relief of symptoms, particularly sore throat. Corticosteroids may reduce the severity and duration of pain, but corticosteroids alone should not be administered as routine treatment, owing to potential side effects [8-10]. Nonsteroidal anti-inflammatory drugs can reduce sore throat pain in 24 hours but are associated with gastrointestinal, renal, or hepatic adverse effects [11]. Although gargles or lozenges with local anaesthetic also provide sore throat pain relief, the effect is short-lived or temporary $[3,12]$.

Owing to a lack of proven effective and safe therapies in Western medicine, an increasing number of patients turn to complementary and alternative medicine [13-15], including traditional Chinese medicine, for relief of symptoms of acute pharyngitis. In traditional Chinese medicinal theory, acute pharyngitis falls into the disease category of pharyngitis (houbi in Chinese Pinyin), and is mainly caused by external causes, including wind and heat. Based on traditional Chinese medicinal clinical practice, windheat syndrome and lung-stomach excess-heat syndrome are the most common syndromes. Of these, lung-stomach excess-heat syndrome is more common in patients, with underlying heat in the lungs and stomach, and is primarily characterized by sore throat, cough, dryness of throat, thirst, and fever. The general traditional Chinese medicinal therapeutic principle is to clear away heat and toxins, and to relieve the sore throat.

Although traditional Chinese medicine has been widely used in China for the treatment of acute pharyngitis for thousands of years, Chinese herbal medicine is not recommended for use as an effective therapy, owing to the lack of high-quality clinical trials in this area [16]. Qi-Wei-Qing-Yan aerosol (QWQYA) is a registered herbal prescription in China and manufactured by Shandong Bencao Pharmaceutical Co., Ltd (Shandong, China). Table 1 lists the detailed formula. Despite the use of QWQYA in acute pharyngitis for several years, its efficacy has never been investigated through a placebocontrolled trial. Moreover, dichlorodifluoromethane (CFC-12), the previous propellant of QWQYA, has gradually been banned by the Ministry of Environmental Protection of the People's Republic of China, owing to its ozone-depleting properties [17]. Alternatively, 1,1,1,2-tetrafluoroethane (HFC-134a) is used as the current aerosol propellant, owing to its negligible ozone depletion potential [18]. Accordingly, the safety of QWQYA with the current aerosol propellant remains unknown.

The objective of this trial is to evaluate the efficacy and safety of two QWQYAs (active medication with the current or previous propellant) in comparison with a placebo aerosol (placebo with the current propellant) in patients with acute pharyngitis (lung-stomach excess-heat syndrome). In this study, the investigators hypothesize that (1) time to complete resolution of sore throat will be shorter in the groups using QWQYA with the current or previous propellant; (2) the proportion of patients who achieve sore throat pain relief will be higher in the groups using QWQYAs; and (3) QWQYA with the current propellant will be safe.

\section{Methods/design}

This is a prospective, multicenter, randomized, doubleblinded, parallel-group, placebo-controlled trial of two QWQYAs (active medication with the current or previous propellant) versus placebo aerosol (placebo with the current propellant) for acute pharyngitis. The study has been authorized by the China State Food and Drug Administration (Approval No. 2014 L00188). In addition, the study was registered with the Chinese Clinical Trial Registry (ChiCTR-IPR-15005991). The trial protocol is conducted in accordance with the Declaration of Helsinki, the code of Good Clinical Practice, and the guidelines of

\begin{tabular}{lll}
\multicolumn{3}{l}{ Table 1 Formula of Qi-Wei-Qing-Yan aerosol } \\
\hline Pinyin name & Botanical or zoological authority & Family name \\
\hline Shandougen & Euchresta japonica Hook. f. ex Regel & Leguminosae \\
Shegan & Belamcanda chinensis (L.) Redouté & Iridaceae \\
Xuanshen & Scrophularia ningpoensis Hemsl. & Scrophulariaceae \\
Maidong & Ophiopogon japonicus (L. f.) Ker-Gawl. & Liliaceae \\
Chansu & Bufo bufo gargarizans Cantor & Bufonidae \\
$\begin{array}{l}\text { Rengong } \\
\text { Shexiang }\end{array}$ & Moschus (Artificial) & Not applicable \\
Bingpian & Dryobalanops aromatica Gaertn.f. & Dipterocarpaceae \\
\hline
\end{tabular}


the International Conference on Harmonisation. Recruitment is scheduled to occur from early April 2015 until October 2015. Figure 1 shows the flow chart of the trial.

\section{Patient population and setting}

The diagnosis is made by medically qualified physician based on the presence of acute onset symptoms and signs suggestive of acute pharyngitis [19]. All patients will be screened for Centor criteria (history of fever $\left(>38{ }^{\circ} \mathrm{C}\right)$, tonsillar exudates, tender anterior cervical adenopathy, and lack of cough) to focus on viral acute pharyngitis. Adults with none or only one of these findings will be included. Traditional Chinese medicinal diagnosis of lung-stomach excess-heat syndrome is based on the Guidelines for Clinical Research of New Chinese Medicine [20]; detailed criteria are presented in Table 2.

A written, signed, and dated informed consent form should be obtained from each patient before any study specific procedures are performed. The participant information sheet and informed consent form will be presented to patients by the responsible clinician, who will explain the exact nature of the study, the implications and constraints of the protocol, the known side effects, and any benefits or risks involved in participation, and the importance of completing the study. It is clearly stated that the participant is free to withdraw from the study at any time for any reason without prejudice to future care, and with no obligation to give the reason for withdrawal.

A total of 420 patients of either sex, aged 18-65 years, will be enrolled from seven study sites across China: (1) West China Hospital of Sichuan University, (2) Xiyuan Hospital of China Academy of Chinese Medical Sciences, (3) the first Affiliated Hospital of Guiyang College of Traditional Chinese Medicine, (4) Ruikang Hospital Affiliated to Guangxi University of Chinese Medicine, (5) the Affiliated Hospital of Shanghai University of Traditional Chinese Medicine, (6) the Affiliated Hospital to Liaoning University of Traditional Chinese Medicine, and (7) the Affiliated Hospital of Chengdu University of Traditional Chinese Medicine. A research assistant in each center will recruit patients either from outpatient departments, or through advertisements in community hospitals or pharmacies. Each participating center will recruit 60 patients.

\section{Inclusion criteria}

1. Clinical diagnosis of acute pharyngitis;

2. Lung-stomach excess-heat syndrome in traditional Chinese medicine;

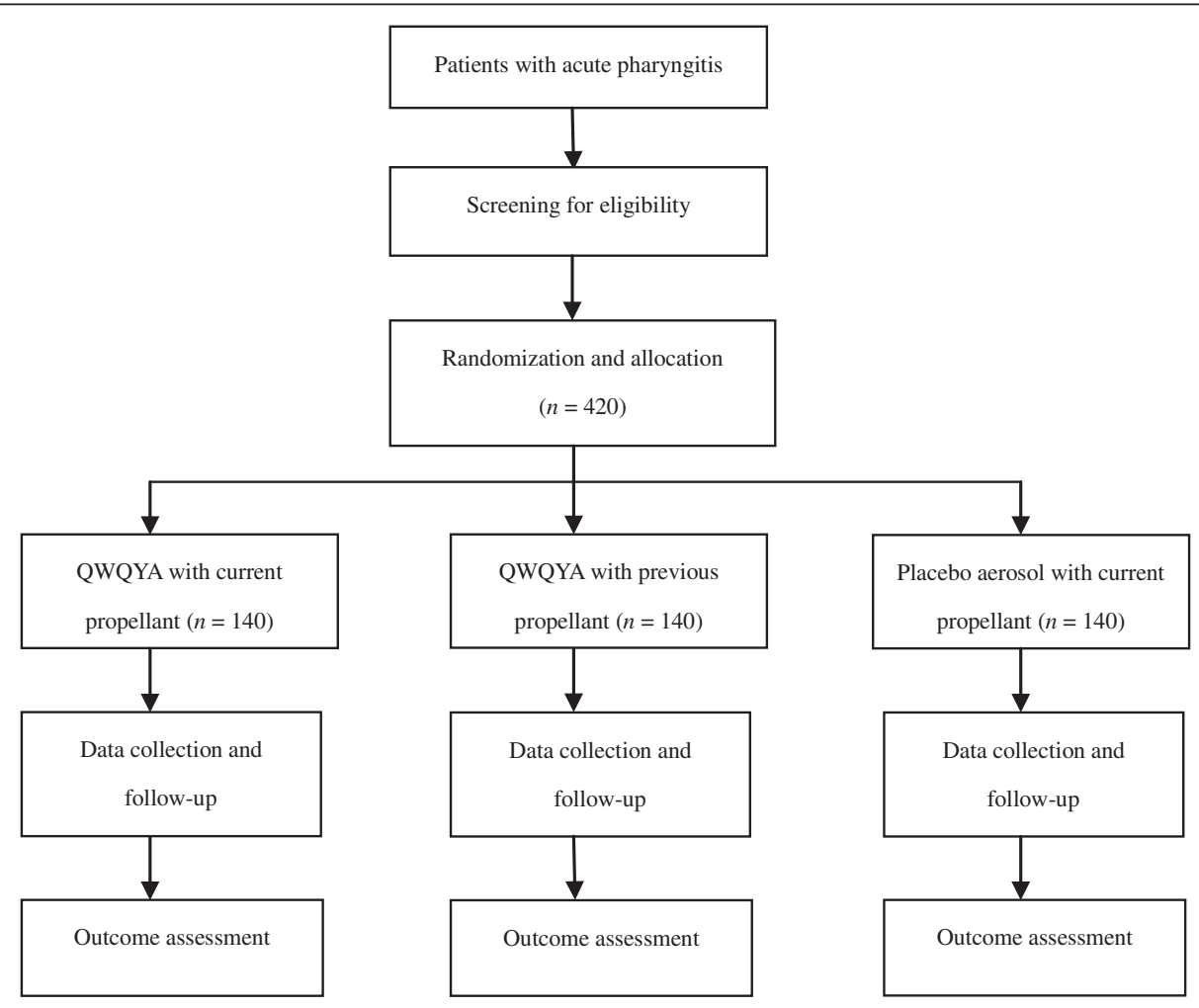

Fig. 1 Study flow chart. QWQYA Qi-Wei-Qing-Yan aerosol 
Table 2 Traditional Chinese medicine syndrome diagnostic criteria for lung-stomach excess-heat syndrome

\begin{tabular}{ll}
\hline Category & Symptoms or signs \\
\hline Main symptom & Sore throat \\
Minor symptoms & $\begin{array}{l}\text { Cough, dryness of throat, thirst, fever, } \\
\text { dry stool, deep-colored urine }\end{array}$ \\
Tongue appearance & Light red appearance \\
Tongue coating & Thin yellow coating \\
Pulse & Rapid and powerful pulse \\
\hline
\end{tabular}

The syndrome can be diagnosed by the presence of the main symptom and $\geq 3$ minor symptoms.

3. Within 48 hours of onset;

4. Centor criteria $\leq 1$;

5. Baseline sore throat score by visual analog scale of 5 or more, and by traditional Chinese medicine score of 6 or more;

6. Aged 18 to 65 years;

7. Patient must voluntarily give written, informed consent and report adverse events and concomitant medication for duration of study.

\section{Exclusion criteria}

1. Patients with temperature greater than $38.5^{\circ} \mathrm{C}$;

2. Use of any medication taken for relief of sore throat prior to study initiation;

3. Current use of analgesic or anti-inflammatory regimen, such as analgesics, nonsteroidal anti-inflammatory drugs, or steroids;

4. Chronic pharyngitis;

5. Patients with any suspected or known heart disease;

6. Sore throat caused by local irritation of mucous membranes due to gastroesophageal reflux or ingestion of caustic substances;

7. Patient with severe primary disease of pulmonary, hepatic, renal, or hematological system, or other serious diseases affecting survival, such as cancer or AIDS;

8. Abnormal results to myocardial markers test, electrocardiogram abnormality, alanine transaminase or aspartate aminotransferase $>1.5$ times of normal upper limit, abnormality of serum creatine, white blood cell count $<3 \times 10^{9} / 1$ or $>10 \times 10^{9} / 1$, and neutrophil granulocyte $>80 \%$;

9. Pregnancy or potential pregnancy or lactation;

10. Allergic constitution or known allergy to any component in QWQYA;

11. Patients taking similar medicines in the previous month or having participated or participating in other trials in previous 3 months;

12. Mentally or legally disabled patients.

\section{Withdrawal criteria}

Once randomly allocated to a group, a patient will be regarded as included in the study. However, patients may voluntarily withdraw from the study at any time for lack of efficacy, worsening condition, or any other reason. Researchers can withdraw patients from the trial owing to patients' lost to follow-up, development of allergic reaction, serious adverse events, condition apparently worsening, use of forbidden medications or treatments, unblinding, or actual usage of trial medication of $<80 \%$ or $>120 \%$ of the required dosage. Withdrawn patients will not be replaced. Any participant will be perceived as having completed the study if all posttreatment assessments have been performed.

\section{Termination criteria}

The entire study will be terminated prematurely or suspended by the investigators in the case of serious adverse events, poor efficacy observed during the study, or significant deviation from the protocol, by the National Pharmaceutical Supervisory and Administrative Department for any reason, or by the sponsor due to management or funding problems.

\section{Randomization and blinding}

Stratified blocked randomization will be applied in the study. Randomization sequence is generated in blocks of seven in a 1:1:1 ratio by an independent statistician not involved with the participants' enrollment or study analysis, using Statistical Analysis System SAS (SAS 9.3). The random treatment assignment for each patient will be concealed and stored in advance in two opaque, sealed, sequentially numbered envelopes. Each set of envelopes corresponds to a unique allocation code containing the specified treatment group. The sealed envelopes are kept in a fireproof place, and will only be opened in case of a medical emergency. Participants, investigators, coordinators together with statisticians are all blinded to group assignments throughout the study.

\section{Investigational medicinal product}

Both QWQYAs contain $6.2 \mathrm{~g} / 7 \mathrm{ml}$ of the active solution with the excipients of ethanol, propylene glycol, and span-80 (one with the current propellant and the other with the previous propellant). Placebo aerosol with the current propellant contains $0.31 \mathrm{~g} / 7 \mathrm{ml}$ of placebo solution with the same excipients for appropriate blinding. Each spray equals $32.6 \mathrm{mg} / 0.037 \mathrm{ml}$ of active solution and $1.6 \mathrm{mg} / 0.037 \mathrm{ml}$ of placebo solution.

Both active and placebo aerosols are manufactured by Shandong Bencao Pharmaceutical Co., Ltd., in accordance with the principles of Good Manufacturing Practice. Medication supplies are coded, packed, and labelled in a blinding manner according to the randomization 
list. Each investigational medicinal product package contains an aerosol. Both active and placebo aerosols are issued in bottles identical in shape, size, and color. A clearly visible label on each package states 'FOR TRIAL USE ONLY' and other information, including function and indication, name, dose, dosing schedule, storage condition, expiry date, and the manufacturer's name. An independent drug administrator in each center is responsible for receipt, handling, storage, dispensing, and retrieval of all investigational medicinal products. The standard operating procedures, and the manner in which the records are kept, must be documented. An overview of consent, screening, enrollment, intervention, timing of measurements, and data collection is shown in Fig. 2.

\section{Interventions}

All participants are randomly allocated to one of three parallel treatment groups: (1) QWQYA with the current propellant, three sprays into the pharyngeal region thrice daily; (2) QWQYA with the previous propellant, three sprays into the pharyngeal region thrice daily; and (3) the placebo aerosol, three sprays into the pharyngeal region thrice daily. The scheduled treatment duration will be 5 consecutive days.

\section{Concomitant treatments and forbidden medication}

Patients can take any Western medication or other Chinese herbal medicine that may relieve sore throat or other symptoms of acute pharyngitis, or use antibiotics and anti-viral agents during the study, but then they will be withdrawn from the study. Medications used to control underlying conditions, such as hypertension or diabetic mellitus, are allowed. Any concomitant treatment or medication administered must be recorded carefully in case report forms, including the name, dose, dosing schedule, mode of administration, and treatment period.

\section{Drug adherence and compliance}

Drug adherence is expected to be good, owing to the short study duration of 5 days and three visits after recruitment. Measures taken to maximize adherence include careful screening, fully informing patients of the potential benefits and risks, and attentive follow-up. Compliance will be checked by patients' documented daily diaries, reporting how many sprays and times they apply the study medication, and by weighing each dispensed aerosol on the date on which the study medication is issued, and again on the date on which it is returned by the patient.

\section{Outcome measures}

\section{Primary outcome measures}

1. Time to complete resolution of sore throat, i.e., length of time from the start of treatment until complete resolution. Sore throat will be assessed using an 11-point (0 to 10) numeric visual analog scale, with 0 indicating 'no pain' and 10 indicating 'worst possible pain'. This score will be evaluated thrice daily, immediately before spraying. Complete resolution of sore throat is defined as a reduction of the score to 1 or less, lasting for more than 24 hours [21].

2. Relief rate of sore throat: this is defined as a reduction by $50 \%$ in the baseline visual analog scale score recorded prior to treatment (day 0), lasting for more than 24 hours. The relief rate of sore throat is the proportion of patients who achieve relief of sore throat.

\section{Secondary outcome measures}

1. Resolution rate of sore throat: proportion of patients who achieve complete resolution of sore throat.

2. Time to relief of sore throat: length of time from the start of treatment until pain relief.

3. Change of intensity of sore throat: assessed by visual analog scale from baseline to post-treatment.

4. Change of traditional Chinese medicine syndrome score from baseline to post-treatment: each traditional Chinese medicine symptom is graded (Fig. 3), and the traditional Chinese medicine syndrome score is the accumulated score of the main symptom score and all minor symptom scores.

5. Change of clinical signs score.

Three primary clinical signs in acute pharyngitis will be observed and recorded before and after treatment. The detailed scoring criteria are shown in Fig. 3.

\section{Safety outcome measures}

Safety outcome measures include monitoring of adverse events, as well as clinical and laboratory findings. In addition to routine tests of blood, urine, and stool, hepatic and renal functions, and myocardial markers, electrocardiography will also be performed at baseline and again after treatment, to assess safety for each group. Patients will be required to record any unexpected symptoms or signs during the treatment period.

\section{Data management, monitoring, and auditing}

A paper case report form will be used to collect data. To ensure maximum follow-up evaluation, the following measures will be taken:

1. Train investigators and study staff in the importance of keeping participants in the trial until the end. 


\begin{tabular}{|c|c|c|c|c|c|c|}
\hline \multirow[b]{3}{*}{ Visit } & \multicolumn{6}{|c|}{ Study period } \\
\hline & \multirow{2}{*}{$\begin{array}{c}\text { Screening } \\
\text { Visit } 0\end{array}$} & \multirow{2}{*}{$\begin{array}{c}\text { Allocation } \\
\text { Visit } 1\end{array}$} & \multicolumn{3}{|c|}{ Post-allocation } & \multirow{2}{*}{$\frac{\text { Closeout }}{\text { Visit } 3}$} \\
\hline & & & & Visit 2 & & \\
\hline Time point & Day -0.5 & Day 0 & Day 1-2 & Day 3 & Day 4-5 & Day 6 \\
\hline \multicolumn{7}{|l|}{ Enrollment } \\
\hline Informed consent & $x$ & & & & & \\
\hline Demographics & $x$ & & & & & \\
\hline Medical history & $x$ & & & & & \\
\hline Concomitant conditions and treatment & $x$ & & & $x$ & & $x$ \\
\hline Current treatment & $x$ & & & $x$ & & $x$ \\
\hline Physical exam and vital signs & $x$ & $x$ & & $x$ & & $x$ \\
\hline Randomization and allocation & & $\times$ & & & & \\
\hline \multicolumn{7}{|l|}{ Interventions } \\
\hline QWQYA with current propellant & & & $\curvearrowright$ & & $\bullet$ & \\
\hline QWQYA with previous propellant & & & $\diamond$ & & $\rightarrow$ & \\
\hline Placebo aerosol with current propellant & & & $\diamond$ & & $\rightarrow$ & \\
\hline \multicolumn{7}{|l|}{ Assessments } \\
\hline Sore throat VAS score & $x$ & $x$ & & $x$ & & $x$ \\
\hline TCM symptoms score & $x$ & $x$ & & $\times$ & & $x$ \\
\hline Clinical signs score & $x$ & $\times$ & & $\times$ & & $x$ \\
\hline Myocardial markers test & $x$ & & & & & $x$ \\
\hline Routine blood / urine / stool test & $x$ & & & & & $x$ \\
\hline Hepatic function test & $\times$ & & & & & $\times$ \\
\hline Renal function test & $x$ & & & & & $x$ \\
\hline Electrocardiogram & $x$ & & & & & $x$ \\
\hline $\begin{array}{l}\text { Chest X-ray (if cough with phlegm and } \\
\text { fever) }\end{array}$ & $x$ & & & & & \\
\hline $\begin{array}{l}\text { Urine pregnancy test (woman of } \\
\text { child-bearing potential) }\end{array}$ & $\times$ & & & & & \\
\hline Adverse events & & & $x$ & $x$ & $x$ & $x$ \\
\hline Drug adherence & & & $x$ & $x$ & $x$ & $x$ \\
\hline
\end{tabular}

Fig. 2 Schedule of study procedures. Participants will receive the study medication for 5 days after enrollment and allocation. The time-points of assessments are shown in the schedule. QWQYA, Qi-Wei-Qing-Yan Aerosol; TCM, traditional Chinese medicine; VAS, visual analog scale

2. Ensure that participants completely understand the research objectives and importance of completing the study.

3. Keep contact information for participants including phone numbers, email addresses, and mailing addresses.

4. Remind participants of their appointments.
5. Make follow-up appointments at each participant's convenience.

6. Provide a remuneration of Chinese $¥ 200$ after completing the study.

7. Provide continued access to effective treatments after the trial for patients whose symptoms are not yet cured. 


\begin{tabular}{|c|c|c|c|c|}
\hline \multirow{2}{*}{ TCM main symptom } & \multicolumn{4}{|c|}{ Score grading } \\
\hline & 0 & 3 & 6 & 9 \\
\hline Sore throat & Not at all & $\begin{array}{l}\text { Mild pain, } \\
\text { without odynophagia }\end{array}$ & $\begin{array}{l}\text { Moderate pain, } \\
\text { with odynophagia }\end{array}$ & $\begin{array}{l}\text { Severe pain, } \\
\text { disturbing regular sleep }\end{array}$ \\
\hline \multirow{2}{*}{ TCM minor symptoms } & \multicolumn{4}{|c|}{ Score grading } \\
\hline & 0 & 1 & 2 & 3 \\
\hline Cough & Not at all & Mild and occasional & Moderate & Severe \\
\hline Dryness of throat & Not at all & Infrequent & Occasional & Frequent and lasting \\
\hline Fever & Not at all & $\begin{array}{l}\text { Conscious fever, } \\
<37.3^{\circ} \mathrm{C}\end{array}$ & $37.3-37.9^{\circ} \mathrm{C}$ & $38.0-38.5^{\circ} \mathrm{C}$ \\
\hline Thirst & Not at all & Mild & Moderate & $\begin{array}{l}\text { Severe; } \\
\text { drinking more water }\end{array}$ \\
\hline Dry stool & Not at all & $\begin{array}{l}\text { Dry stool, } \\
\text { once a day }\end{array}$ & $\begin{array}{l}\text { Constipation, twice to } \\
\text { three times a day }\end{array}$ & $\begin{array}{l}\text { Constipation and abdominal } \\
\text { distension, need cathartics to expel }\end{array}$ \\
\hline Deep-colored urine & Not at all & Yellow urine & $\begin{array}{l}\text { Yellow and red urine, } \\
\text { small amount }\end{array}$ & $\begin{array}{l}\text { Brown urine, buming sensation } \\
\text { during urination }\end{array}$ \\
\hline \multirow{2}{*}{ Clinical signs } & \multicolumn{4}{|c|}{ Score grading } \\
\hline & 0 & 1 & 2 & 3 \\
\hline Pharyngeal hyperemia & Not at all & Mild & Moderate & Severe with swelling \\
\hline $\begin{array}{l}\text { Retropharyneal congestion } \\
\text { and swelling }\end{array}$ & Not at all & Mild & Moderate & Severe \\
\hline $\begin{array}{l}\text { Mandibular angle lymph } \\
\text { node tendemess }\end{array}$ & Not at all & Mild & Moderate & Severe \\
\hline
\end{tabular}

Fig. 3 Symptoms and signs score. TCM, traditional Chinese medicine

8. Make efforts to obtain the participant's consent for the collection of data outcomes when medication discontinuation occurs.

After completion of data collection, double data entry will be performed to transcribe the data into the computer database as soon as possible after recruitment and follow-up evaluation so that the patient and data collector are still available if responses are found to be missing or out of range. All information on the patients and outcome variables will be stored, updated, and monitored using EpiData3.1, which will also be used to format the data for statistical analysis. Regular backups and off-site storage will be conducted, to prevent loss of the database. The principal investigator, data managers, sponsor, and statisticians will confirm and lock the database prior to data processing. Only data managers and statisticians will have access to the final data. Original case report forms and any other records will be kept on file at each study site for 5 years.
Interim analyses will not be performed, as the investigational product is a registered and clinically used aerosol and the study is scheduled to last for approximately 6 months. An independent data monitoring committee will be set up to monitor patient safety and treatment efficacy data. Moreover, a monthly progress report on enrollment, recruitment, study participations, safety, and completion will be issued to the principal investigator. The regulatory authority at each study site and auditors independent of the investigators and sponsor will conduct a monthly audit, including, but not limited to, trial procedure and compliance with the protocol, standard operating procedures, code of Good Clinical Practice, and the applicable regulatory requirements. Any observation or finding should be documented.

\section{Adverse event reporting}

An adverse event will be defined as any unfavorable and unintended sign (including abnormal laboratory findings), symptom, or disease temporally associated with 
the use of the study medication. All adverse events will be recorded in detail, including event, date of occurrence, grade, whether expected or unexpected, date resolved, and treatment patients receive specifically related to the event, and will be categorized as clearly not, doubtfully, may be, likely, or clearly related to the study medication. A serious adverse event will be defined as any untoward medical occurrence that, at any dose, results in death, is life-threatening, requires inpatient hospitalization or prolongation of existing hospitalization, results in persistent or significant disability or incapacity, is a congenital anomaly or birth defect, or other important medical event. Other events that are not likely to result in death, are not life-threatening, or do not require hospitalization, might be considered as serious adverse event if the event might jeopardize the patient and might require medical or surgical intervention to prevent one of the outcomes listed above.

Medically qualified investigators will record, assess, and report adverse events. Non-serious adverse events will be recorded in case report forms, and listed in a monthly progress report to the principal investigator and sponsor. Serious adverse events must be reported to the institutional review board, principal investigator, sponsor, and the Drug Registration Division of China's State Food and Drug Administration and Research within 24 hours. The blinding procedures will be broken in case of a medical emergency.

\section{Sample size calculation}

The sample size was calculated on the basis of the two primary outcomes, using 'Non-inferiority tests for two proportions [differences]' in PASS 11.0. Based on a previous study [22], the mean time to complete resolution of sore throat in both active treatments was similar, both with a standard deviation of 2.2 days. A difference of 1 day or more between groups was regarded a clinically important effect. Setting a one-sided $\alpha$ of 0.05 and $\beta$ of 0.1 , the sample size was estimated to be 84 cases for each group. Based on consultation with clinical experts and a preliminary unpublished study provided by the sponsor, the relief rate of sore throat in QWQYA with previous propellant was estimated at $91 \%$, with a difference of $10 \%$ or more considered clinically significant. The sample size was estimated at 118 participants for each group, assuming a one-sided $\alpha$ of 0.05 and $\beta$ of 0.15 . Both the sample sizes were calculated according to the formula:

$$
n=\left[\frac{2\left(Z_{1-\alpha / 2}+Z_{\beta}\right) \sigma}{\delta-\Delta}\right]^{2}
$$

Therefore, the final sample size for each group was 118 participants. Taking approximate dropout rates of $20 \%$ into account, the number of participants to be recruited was estimated at 140 per group. Therefore, a total of 420 patients will be recruited in this trial.

\section{Statistical analysis}

An independent statistician will perform statistical analysis using SAS 9.3. The full analysis set population includes all randomized participants as allocated. Only missing data regarding the primary outcomes will be adjusted using the last observation carried forward method. The per-protocol population includes only patients who fulfil the protocol. Analysis of efficacy will be performed based on the full analysis set and per-protocol population. Safety analyses will be performed in those populations, which include all randomized participants who receive at least one spray of medication and who have at least one visit for safety, whether or not they are withdrawn prematurely.

A fully specified statistical analysis plan will be written before unmasking. Quantitative variables will be described as mean \pm standard deviation or median, and qualitative variables as frequency and percentage. Baseline analyses will be performed using analysis of variance (ANOVA) or rank-sum test for quantitative data and chi-squared or Fisher's exact test for qualitative data. A non-inferiority test will be used to compare the two active QWQYA groups, and a superiority test to compare the current-propellant QWQYA group and the placebo group. A one-sided confidence interval method will be used in efficacy analyses. Statistical significance will be considered for $P \leq 0.05$.

\section{Ethical considerations and dissemination}

The study protocol has been reviewed and approved by the Biomedical Ethics Committee of West China Hospital of Sichuan University (Chengdu, China). Any modifications, including the principal investigator, study protocol, and informed consent forms must be re-submitted, reviewed, and approved by the Biomedical Ethics Committee. Trained research investigators will explain the study procedures using information sheets, which will be provided to all potential participants prior to participation. All participants must voluntarily give their written, informed consent prior to any study procedures. Participants with impaired decision-making capacity who might have difficulty weighing the risks and benefits of the study will be excluded from this study. The principal investigator, sponsor, ethical committees, and drug regulation agencies have access to individual patient's data, and 
no ancillary studies will use these participants' data. Moreover, each patient will be identified with a unique identity without any other personal identifier for purposes of maintaining confidentiality. This study protocol has been prepared according to the SPIRIT checklist [23].

Additionally, chest X-rays will be obtained in selecting eligible participants, to exclude patients with lower respiratory tract infection, and a urine pregnancy test (strip) will be used to detect early suspected or unknown pregnancy in women with child-bearing potential.

A preliminary study (an unpublished observation by Professor Jia Miao) suggests that QWQYA might be associated with a risk of mild reduction of heart rate within normal range. However, another two trials $[24,25]$ did not find any such association. In this study, myocardial markers will be tested and electrocardiography will be performed in potential participants, and a detailed history will be taken, to exclude those with an underlying heart disease. In addition, the test of myocardial markers and electrocardiography will be performed again after treatment to monitor participants for potential adverse effects.

The results of this study will be presented at national or international conferences and submitted to peerreviewed journals.

\section{Discussion}

A Cochrane review [16] of 12 randomized controlled trials that assessed the efficacy and safety of Chinese herbal medicine for sore throat found that trials in this area were methodologically of poor quality, with controversial and questionable evidence of efficacy. Moreover, the randomized controlled trials included in this review and trials conducted thereafter [26-28] all used a 'positive effect drug' as a control, comparing the Chinese herbal medicine under investigation with another Chinese herbal medicine or a Western medicine. However, the efficacy of these 'positive effect drugs' has not yet been well established. To the best of our knowledge, this will be the first multicenter, double-blind, randomized, placebo-controlled trial of Chinese herbal medicine designed to treat acute pharyngitis in an adult population. Not only might it establish the basis for the efficacy and safety of QWQYA in the treatment of acute pharyngitis, but it might also provide evidence regarding Chinese herbal medicine for treating acute pharyngitis and an alternative treatment option for the management of acute pharyngitis.

This trial is placebo-controlled and focuses on viral pharyngitis in adults. Although it is not always easy to distinguish viral from bacterial causes, laboratory confirmation of bacteria, including a rapid antigen detection test and throat culture is not indicated and recommended in adults with a lower likelihood of GABHS infection [29]. In this trial, Centor criteria (a history of fever, tonsillar exudates, tender anterior cervical adenopathy, and lack of cough) will be applied to exclude patients with potential GABHS pharyngitis, with the absence of three or four of the criteria indicating a negative predictive value of more than $80 \%[30,31]$.

It is well-known that the gold standard for assessing the efficacy of specific intervention is a randomized placebo-controlled trial. Ideally, a placebo should be an inactive substance that is pharmacodynamically inert. However, it is significantly difficult to develop appropriate placebos, in terms of smell, taste, and color, in clinical trials of herbal medicine especially those of compound formula in a liquid form. To our knowledge, no validated placebo of Chinese herbal medicine has been developed in the setting of acute pharyngitis. Moreover, several clinical trials outside the setting of acute pharyngitis have applied 10-20 times dilutions of active herbs as placebos in China and Japan [32, 33]. In this trial, one-twentieth of the total dose of active solution is used as the placebo, since it is assumed to be unlikely that a tiny amount of $14.4 \mathrm{mg}$ per day could be an effective treatment. Both the active aerosols and the placebo aerosol are issued in bottles identical in size, shape, and color, to ensure appropriate blinding. In addition, participants are required not to communicate with each other, to minimize the possibility of revealing differences between the placebo and active medication.

\section{Trial status}

This trial is preparing for patient recruitment at the time of manuscript submission.

\section{Abbreviations}

ANOVA: analysis of variance; GABHS: Group A beta-hemolytic streptococcal; QWQYA: Qi-Wei-Qing-Yan aerosol.

\section{Competing interests}

The authors declare that they have no competing interests.

\section{Authors' contributions}

HLJ participated in the design and development of the study protocol, and drafted the initial manuscript. WL participated in the design and development of the study protocol, and helped to revise the manuscript. BS participated in the design and development of the study protocol. JYZ participated in the study design, and performed the sample size calculation and statistical analysis plan. BM conceived the study, participated in the design and development of the study protocol, helped to revise the manuscript, and was responsible for coordination. All authors have read and approved the final manuscript.

\section{Acknowledgements}

The study is financially supported by Shandong Bencao Pharmaceutical Co., Ltd. (18 Xiguan Street, Zichuan Road, Zibo, Shandong Province, China), which provided the QWQYA and placebo aerosols. However, the funder has no input into the study design, protocol preparation, or future data analysis 
and interpretation. We also express our gratitude to Methods in Epidemiologic, Clinical and Operations Research (MECOR) program (China 2015), developed by the American Thoracic Society for guidance regarding clinical trial protocol.

\section{Author details}

${ }^{1}$ Department of Integrated Traditional Chinese and Western Medicine, West China Hospital, Sichuan University, No. 37 Guoxue Street, Chengdu 610041, Sichuan Province, China. ${ }^{2}$ Department of Epidemiology and Biostatistics, School of Public Health, Sichuan University, No. 16 People's South Road, Chengdu 610041, Sichuan Province, China.

Received: 31 March 2015 Accepted: 5 February 2016

Published online: 19 February 2016

\section{References}

1. Hing E, Hall MJ, Xu J. National Hospital Ambulatory Medical Care Survey: 2006 outpatient department summary. Natl Health Stat Report. 2008; 1-31.

2. Wei $J$, Kasperbauer $J$, Weaver AL, Boggust AJ. Efficacy of single-dose dexamethasone as adjuvant therapy for acute pharyngitis. Laryngoscope. 2002;112:87-93.

3. Bisno AL. Acute pharyngitis. N Engl J Med. 2001;344:205-11.

4. Centor RM, Atkinson TP, Ratliff AE, Xiao L, Crabb DM, Estrada CA, et al. The clinical presentation of fusobacterium-positive and streptococcal-positive pharyngitis in a university health clinic: a cross-sectional study. Ann Intern Med. 2015;162:241-7.

5. Spinks A, Glasziou PP, Del Mar CB. Antibiotics for sore throat. Cochrane Database Syst Rev. 2013;11:CD000023.

6. Barnett ML, Linder JA. Antibiotic prescribing to adults with sore throat in the United States, 1997-2010. JAMA Intern Med. 2014;174:138-40.

7. Gulliford MC, Dregan A, Moore MV, Ashworth M, Staa T, McCann G, et al. Continued high rates of antibiotic prescribing to adults with respiratory tract infection: survey of 568 UK general practices. BMJ Open. 2014;4:e006245.

8. Korb K, Scherer M, Chenot JF. Steroids as adjuvant therapy for acute pharyngitis in ambulatory patients: a systematic review. Ann Fam Med. 2010;8:58-63.

9. Wing A, Villa-Roel C, Yeh B, Eskin B, Buckingham J, Rowe BH. Effectiveness of corticosteroid treatment in acute pharyngitis: a systematic review of the literature. Acad Emerg Med. 2010:17:476-83.

10. Hayward G, Thompson M, Heneghan C, Perera R, Del Mar C, Glasziou P. Corticosteroids for pain relief in sore throat: systematic review and meta-analysis. BMJ. 2009;339:b2976.

11. Moore N, Charlesworth A, Van Ganse E, LeParc JM, Jones JK, Wall R, et al. Risk factors for adverse events in analgesic drug users: results from the PAIN study. Pharmacoepidemiol Drug Saf. 2003;12:601-10.

12. Chenot JF, Weber P, Friede T. Efficacy of ambroxol lozenges for pharyngitis: a meta-analysis. BMC Fam Pract. 2014;15:45.

13. Hubbert M, Sievers $H$, Lehnfeld R, Kehrl W. Efficacy and tolerability of a spray with Salvia officinalis in the treatment of acute pharyngitis - a randomised, double-blind, placebo-controlled study with adaptive design and interim analysis. Eur J Med Res. 2006;11:20-6.

14. Saxena RC, Singh R, Kumar P, Yadav SC, Negi MP, Saxena VS, et al. A randomized double blind placebo controlled clinical evaluation of extract of Andrographis paniculata (KalmCold) in patients with uncomplicated upper respiratory tract infection. Phytomedicine. 2010;17:178-85.

15. Brinckmann J, Sigwart $H$, van Houten Taylor L. Safety and efficacy of a traditional herbal medicine (Throat Coat) in symptomatic temporary relief of pain in patients with acute pharyngitis: a multicenter, prospective randomized, double-blinded, placebo-controlled study. J Altern Complement Med. 2003;9:285-98.

16. Huang $Y$, Wu T, Zeng L, Li S. Chinese medicinal herbs for sore throat. Cochrane Database Syst Rev. 2012;3:CD004877.

17. Decree of the State Council of the People's Republic of China (No. 573): Regulations on administration of ozone depleting substances. http://www. gov.cn/flfg/2010-04/16/content_1594673.htm. Accessed 16 April 2010.

18. Ravishankara AR, Turnipseed AA, Jensen NR, Barone S, Mills M, Howard CJ, et al. Do hydrofluorocarbons destroy stratospheric ozone? Science. 1994;263:71-5.

19. Tian YQ. Otolaryngology head and neck surgery. 8th ed. Beijing: People's Health Publishing House; 2013.
20. Zheng XY. Guiding principle of clinical research on new drugs of traditional Chinese medicine (trial implementation). Beijing: Chinese Medical Science and Technology Press; 2002.

21. Lindbaek M, Francis N, Cannings-John R, Butler CC, Hjortdahl P. Clinical course of suspected viral sore throat in young adults: cohort study. Scand J Prim Health Care. 2006:24:93-7.

22. Leelarasamee A, Leowattana W, Tobunluepop P, Chub-upakarn S, Artavetakun W, Jarupoonphol $\mathrm{V}$, et al. Amoxicillin for fever and sore throat due to non-exudative pharyngotonsillitis: beneficial or harmful? Int J Infect Dis. $2000 ; 4: 70-4$

23. Chan AW, Tetzlaff JM, Altman DG, Laupacis A, Gøtzsche PC, Krleža-Jerić K, et al. SPIRIT 2013 statement: defining standard protocol items for clinical trials. Ann Intern Med. 2013;158:200-7.

24. Biao W. Effect of QiWeiQingYan aerosol on infantile herpangina of 70 cases. Henan Med Res. 2011:2:34-5.

25. Zhang MR, Bi SK, Zhang MY. QiWeiQingYan aerosol in the treatment of acute pharyngitis of 120 cases. External Treatment Tradit Chin Med. 2000;9:19-20.

26. Liu QF, Sun HB, Quan WJ, Qu ZY. Clinical observation of Huangqin Jingye Jiedu capsule in the treatment of acute pharyngitis. Nei Mongol J Tradit Chin Med. 2013;32:13-4.

27. Wang Y, Lin YP, Deng KB, Liu TX. Clinical study on compound Yejuhua lozenge in treating sore throat (wind-heat). Chin J Inform Tradit Chin Med. 2012;19:6-8.

28. Zhu HX, Chai F, Cao B. Clinical Studies on Zhongsheng pills in treating acute pharyngitis (wind-heat syndrome). Strait Pharm J. 2014;6:68-70.

29. Pelucchi C, Grigoryan L, Galeone C, Esposito S, Huovinen P, Little P, et al. Guideline for the management of acute sore throat. Clin Microbiol Infect. 2012;18 Suppl 1:1-28.

30. Centor RM, Witherspoon JM, Dalton HP, Brody CE, Link K. The diagnosis of strep throat in adults in the emergency room. Med Decis Making. 1981;1:239-46.

31. Aalbers J, O'Brien KK, Chan WS, Falk GA, Teljeur C, Dimitrov BD, et al. Predicting streptococcal pharyngitis in adults in primary care: a systematic review of the diagnostic accuracy of symptoms and signs and validation of the Centor score. BMC Med. 2011;9:67.

32. Wu J. Placebo control and clinical trial of Chinese medicine. Zhong Xi Yi Jie He Xue Bao. 2010;8:906-10

33. Tang XD, Bian LQ, Gao R. Exploration into the preparation of placebos used in Chinese medicinal clinical trial. Zhongguo Zhong Xi Yi Jie He Za Zhi. 2009;29:656-8.

\section{Submit your next manuscript to BioMed Central and we will help you at every step:}

- We accept pre-submission inquiries

- Our selector tool helps you to find the most relevant journal

- We provide round the clock customer support

- Convenient online submission

- Thorough peer review

- Inclusion in PubMed and all major indexing services

- Maximum visibility for your research

Submit your manuscript at www.biomedcentral.com/submit 